\title{
Direct monitoring of metal ion transfer between two trafficking proteins.
}

\author{
Richard Ledwidge, Rebecca Soinski and Susan M. Miller*
}

\section{Stopped-Flow Experiments}

All reactions were performed in single mixing mode of a HiTech SF-61DX2 instrument (now TgK Scientific Ltd., U.K.) with a thermostatted cell chamber connected to a circulating constant temperature water bath set at $25^{\circ} \mathrm{C}$. Fluorescence data were collected in dual beam mode with photomultiplier detection using an excitation wavelength of $456 \mathrm{~nm}$ and a emission filter with a $500 \mathrm{~nm}$ cutoff. Raw fluorescence data were imported into Kaleidagraph (Synergy software) and fit to a model describing a single exponential with the following exception. $\mathrm{In}_{\mathrm{Hg}}{ }^{+2}$ transfer experiments in the forward direction, there was a very small second linear phase $(\sim 0.05 / \mathrm{sec})$ at higher $\mathrm{Hg}-\mathrm{NmerA}$ concentrations. Since the rates and amplitudes of the linear phase were kinetically insignificant, this phase was subtracted and the remaining data refit to a single exponential. Only the single exponential fluorescence data were used in the modeling described below.

\section{Fluorescence pH experiments}

Catalytic core proteins were purified as described in Ledwidge, et al. (1) with one modification. In the final step to remove urea, catalytic core protein is passed through a G-50 column $(1.5 \times 25 \mathrm{~cm})$ equilibrated in $10 \mathrm{mM} \mathrm{KPi}, \mathrm{pH} 6.0$ at $4{ }^{\circ} \mathrm{C}$. A three buffer cocktail was made that had a pH buffering range from $4-$ 11.5 with constant ionic strength (2). The cocktail contained 0.2 M MES, 0.1 M ethanolamine, and 0.1 M triethanolamine. The $\mathrm{pH}$ of the buffer cocktail was made acidic or basic by the addition of concentrated $\mathrm{HCl}$ or $\mathrm{KOH}$. Catalytic core protein $(10 \mu \mathrm{M})$ in $10 \mathrm{mM} \mathrm{KPi}, \mathrm{pH} 6.0$ was mixed in the stopped-flow spectrophotometer with the three buffer cocktail at different $\mathrm{pH}$ values. Data were collected for a few 
seconds until the fluorescence stabilized.

\section{Fluorescence $\mathrm{Hg}^{+2}$ transfer experiments}

The purification of catalytic core and NmerA as well as the formation of Hg-NmerA and $\mathrm{Hg}-\mathrm{CCCC}$

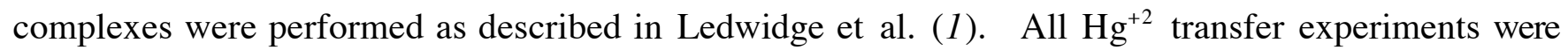
performed with proteins in $50 \mathrm{mM} \mathrm{KPi}, \mathrm{pH}$ 7.3. Pseudo-first order conditions were maintained with catalytic core (reduced or $\mathrm{Hg}^{+2}$-bound) equal to $5 \mu \mathrm{M}$ (final concentration) and $\mathrm{NmerA}\left(\mathrm{Hg}^{+2}\right.$-bound or reduced) at 50, 75, 100, 150, 250, and $500 \mu \mathrm{M}$ (final concentrations).

The observed rate constants, $k_{o b s}$, in both directions showed a hyperbolic dependence on the concentration of reduced or Hg-bound NmerA (Figure 3 in the paper) that could be described by the minimal two-step mechanism in Scheme S1 and eq S1 (3). For the forward reaction, the best fit gave a finite value for $k_{r e v}$, while the best fit for the reverse reaction gave $k_{r e v}=0$.

\section{Scheme S1}

$$
\begin{aligned}
& \mathrm{A}+\mathrm{B} \frac{k_{1}}{k_{2}} \mathrm{C} \underset{k_{r e v}}{\frac{k_{\max }}{k_{\text {max }}}} \mathrm{D} \\
& k_{\text {obs }}=\frac{k_{\max } \times \mathrm{B}}{K_{1 / 2}+\mathrm{B}}+k_{\text {rev }} ; K_{1 / 2}=\frac{k_{2}+k_{\max }}{k_{1}}
\end{aligned}
$$

In this scheme $\mathrm{A}$ represents the appropriate catalytic core protein and B is the appropriate NmerA species. In both cases $\mathrm{A}$ and $\mathrm{C}$ have the same fluorescence properties, and the change in fluorescence occurs upon conversion of $\mathrm{C}$ to $\mathrm{D}$. Because the two reactions define the same transfer reaction in opposing directions, the overall mechanism must be consistent with the combination of these opposing apparent two-step processes. The three step mechanism shown in Scheme 1 and Scheme S2 (below) represents the minimal mechanism that can account for the observed concentration dependences on the fluorescence changes in the two opposing reactions. 


\section{Kinetic modeling}

Berkeley Madonna software (developed by George Oster at the University of California at Berkeley) was used to model the kinetic data according to the three step kinetic scheme below

\section{Scheme S2}
$\mathrm{A}+\mathrm{B} \underset{k_{2}}{\stackrel{k_{1}}{\rightleftharpoons}} \mathrm{C} \stackrel{k_{3}}{\stackrel{k_{4}}{\rightleftharpoons}} \mathrm{D} \underset{k_{6}}{\stackrel{k_{5}}{\rightleftharpoons}} \mathrm{E}+\mathrm{F}$

where $\mathrm{A}=$ catalytic core (partially quenched fluorescence)

$\mathrm{B}=\mathrm{Hg}-\mathrm{Nmer}$ A (No fluorescence)

$\mathrm{C}=$ catalytic core- $\mathrm{Hg}-\mathrm{NmerA}$ complex (species II in Scheme 1, same fluorescence as A)

$\mathrm{D}=$ catalytic core-Hg-NmerA complex (species III in Scheme 1, same fluorescence as E)

$\mathrm{E}=$ catalytic core- $\mathrm{Hg}$ (fully fluorescent)

$\mathrm{F}=$ NmerA (No fluorescence)

In order to model the kinetic data, changes in fluorescence must first be converted into changes in concentration of fluorescent and quenched species. Since all of the reaction traces could be fit to a single exponential, there were no detectable changes in fluorescence associated with formation of " $\mathrm{C}$ " in the forward direction, nor of " $\mathrm{D}$ " in the reverse direction. Thus, it was assumed for this model that species with C558 in the thiolate state (A and C) have the same quenched fluorescence intensity, while those with C558 bound to $\mathrm{Hg}^{+2}$ (D and E) have the same full fluorescence intensity. Using these assumptions along with the starting concentration of catalytic core and NmerA, and the initial, final, and maximal fluorescence changes (the latter measured in the control reaction with $\left.\mathrm{Hg}(\mathrm{Cys})_{2}\right)$, total concentrations of quenched $(\mathrm{A}+$ C) and fluorescent $(D+E)$ species were calculated for the purpose of modeling. We then performed a global fit of the forward and reverse reactions at all concentrations using $k_{\max }$ and $K_{1 / 2}$ values obtained as described above from data in Figure 3 as a guide for assigning initial rate constant estimates. This was performed iteratively until satisfactory fits to the data sets were obtained. Fits to the reverse reaction are shown in Figure S1. The values from the simultaneous modeling that gave the best fits in both directions 
are presented in Scheme 1 in the paper.

As is typical in multiparameter modeling, similarly good fits could be obtained where the absolute values of the individual rate constants are slightly different, but the equilibria and partitioning of intermediates remain the same as those in Scheme 1. One potential error in the relative magnitudes of the rate constants obtained here is the uncertainty in the intrinsic fluorescence of the apparent intermediates in the reaction relative to their respective starting enzyme species. Although the reaction traces provide no evidence for accumulation of intermediates with altered fluorescence properties, the presence of small differences in intrinsic fluorescence would introduce errors in the concentrations of the intermediates calculated using the assumption of identical properties used here, which would propagate into errors in the rate constants that fit the model. One final caveat is that the "intermediates" required by the hyperbolic fits need not be single species, but could in either or both cases be a rapid equilibrium mixture of species whose individual fluorescence properties could vary from those of the starting species, but whose composite fluorescence is essentially equal to that of the starting species. In this case, the rate constants from the fit for the current model would represent observed rate constants for the interconversion of the mixtures.

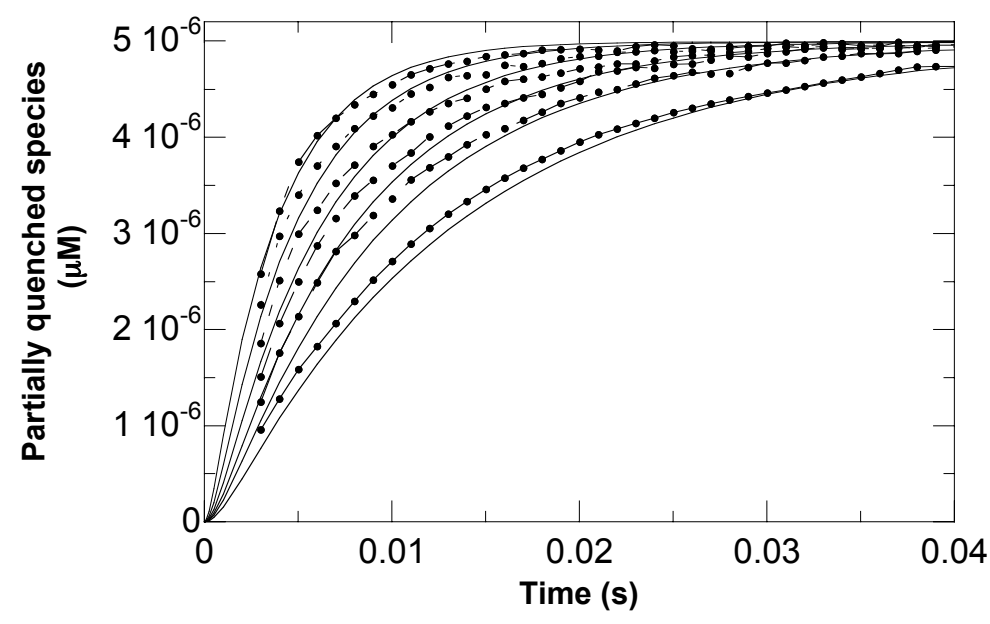

Figure S1. Model fits of $\mathrm{Hg}^{+2}$ transfer in the reverse direction obtained using Berkeley Madonna software. Reaction of $5 \mu \mathrm{M} \mathrm{Hg-CCCC} \mathrm{with} \mathrm{increasing}(50,75,100,150,250$ and $500 \mu \mathrm{M})$ reduced NmerA. Data are shown as connected points; fits are solid lines.

\section{References}


1) Ledwidge, R.; Patel, B.; Dong, A.; Fiedler, D.; Falkowski, M.; Zelikova, J.; Summers, A. O.; Pai, E. F.; Miller, S. M. 2005 (Submitted to Biochemistry).

2) Ellis, K.J.; Morrison, J.F. Meth in Enzymol 1982, 87, 405-26.

3) Strickland, S.; Palmer, G.; Massey, V. J. Biol. Chem. 1975, 250, 4048-52. 